\title{
Family-related risk factors of obesity among preschool children: results from a series of national epidemiological surveys in China
}

\author{
Xin-Nan Zong, Hui Li and Ya-Qin Zhang
}

\begin{abstract}
Background: Family-based child obesity prevention and control strategy has not yet established in many countries or regions, including China, thus what it needs to do now is to continuously develop and improve the strategies. The purpose of this study were to describe a wider spectrum of risk factors of obesity among preschool children and add to the mounting evidence for further improving suggested intervention measures in future family-based programs.

Methods: Data was collected as part of a series of national epidemiological surveys in childhood conducted in 9 Chinese cities. A population-based, 1:1 matched case-control design was employed to examine risk factors of obesity by means of conditional logistic regression. Obesity was defined as the International Obesity Task Force (IOTF) BMI-for-age cut offs. Eligible subjects were 1234 boys and 610 girls aged 3-7 years in 1996 and 2290 boys and 1008 girls in 2006, including obese and non-obese.
\end{abstract}

Results: High birth weight, gestational hypertension and parents' BMI were closely associated with childhood obesity. Breast feeding in the first 4 months was a protective factor in univariate model in 2006 ( $O R=0.834, P=0.0234$ ), but the association was not seen in multivariate. Appetite, eating speed, daily time and intensity for outdoor activities, night sleep time, and time for TV viewing were identified statistically by multivariate model. Those children brought up in extended family or mainly raised by their grandparents or lived in high income or low education families might have an increased risk of becoming obese. Parents' attitudes on weight control of their children significantly differed between obese and non-obese groups.

Discussion: A wider spectrum of risk factors and an empirical aggregation of family-related risk factors are discussed to further improve future family-based child obesity prevention and control strategies. Most of the risk factors identified by this study presented ranked or quantitative characteristics which might be transformed from unhealthy threshold to healthy range by behavior modification. Some variables are likely to interact each other, such as appetite and eating speed, or outdoor activity and TV viewing, or BMI and income, but which needs to be further explored in future surveys.

Conclusions: The family-related risk factors were summarized from our identified risk factors of obesity among preschool children which strongly supported the further development of family-based programs in preschool period.

Keywords: Obesity, Risk factor, Family, Behavior, Child, China

\section{Background}

Global obesity in children has reached epidemic proportions [1] and increased trend of obesity was also documented in Chinese children over the last decades [2]. Contributing factors to the increases in obesity include a decline in positive health behaviors, such as making healthy dietary choices, engaging in physical activity, and limiting sedentary behaviors. Results on 38

\footnotetext{
* Correspondence: huiligrowth@163.com

Department of Growth and Development, Capital Institute of Pediatrics, No.2 Yabao Road, Chaoyang District, Beijing 100020, China
}

published articles of diet, physical activity, and sedentary behaviors showed support for the role of parenting and physical environmental factors [3]. Family and physical environmental contextual factors related to health behaviors are increasingly the focus of health behavior interventions in line with the bioecological model. Family-based program has been used to treat obesity in youth in some countries, but few programs incorporate a theoretical framework for studying a family systems approach in relation to youth health behavior change [4].

Preschool period is not only the time of the adiposity or body mass index rebound, but also a key link between 
infancy and school-age period. School-based programs were proved to be effective in reducing obesity for school children [5-7]. School-based and family-involved obesity intervention program is being tested among children aged 10-12 [8]. As schools are an important setting for health promotion for school-aged children, family environment is regarded as a good setting for preschool children, but family-based obesity prevention strategies in childhood were not paid enough attention and not well implemented in practice.

We have noticed that one systematic review showed the strength of evidence was low to support the effectiveness of family-based child obesity prevention programs [9]. The family-based strategy is however still generally on the primary stage and less well-developed. Family-based strategy has not yet established in many countries or regions, including China [10], thus what it needs to do now is to continuously develop and improve the strategies according to own characteristics. In addition, it remains unclear for the practical operability of suggested intervention measures and the compliance of children, parents and families for existing family-based strategies. The purpose of this study were to describe a wider spectrum of risk factors of obesity among preschool children from a series of national epidemiological surveys in China [11] and add to the mounting evidence for further improving suggested measures in future family-based obesity prevention and control programs in childhood.

\section{Methods}

Data source and subjects

Data were collected as part of the series of the National Epidemiological Survey on Simple Obesity in Childhood (NESSOC) conducted in 9 Chinese cities (Fig. 1) in 1996

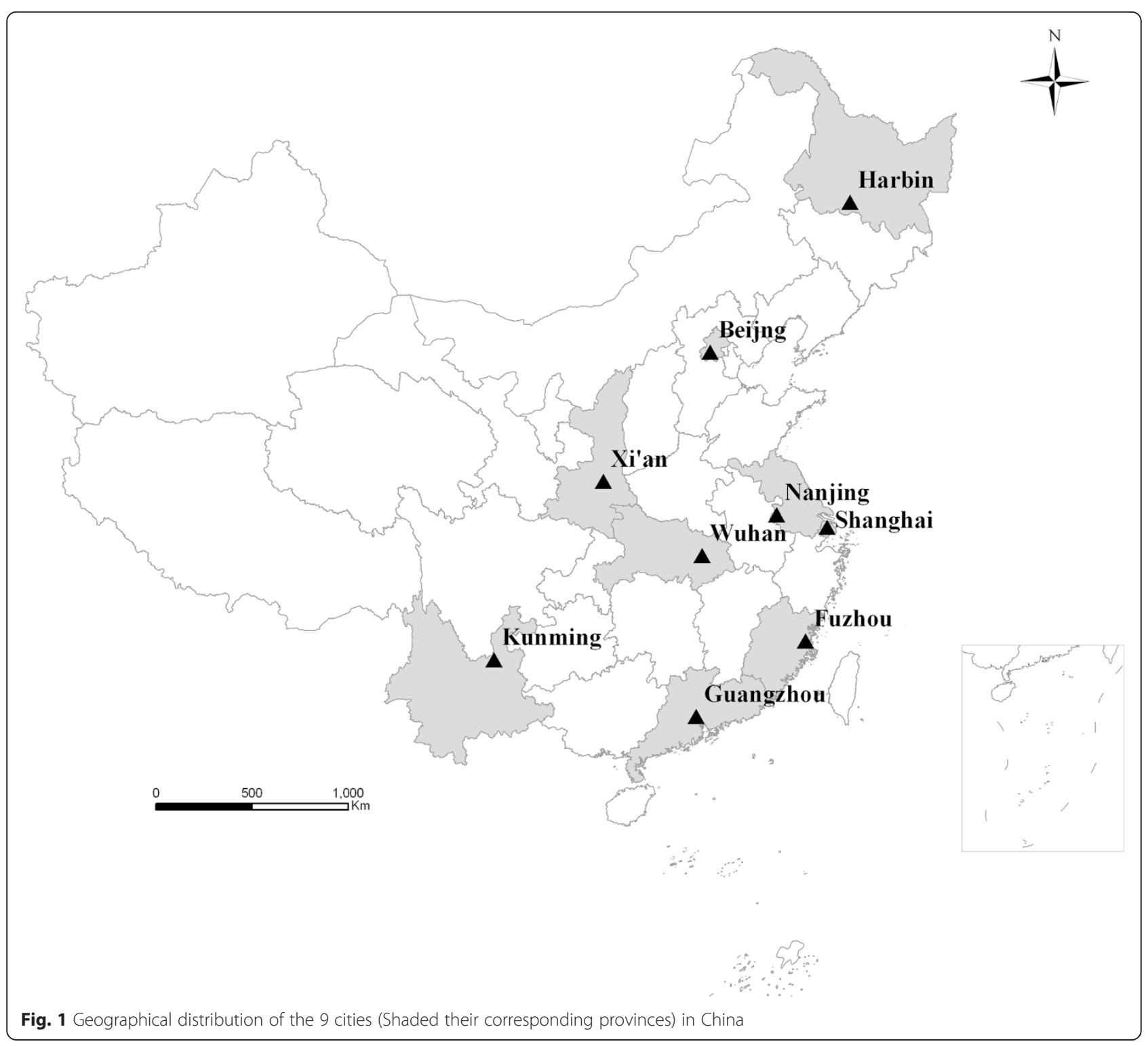


and 2006, respectively. Among the 9 cities, Beijing and Shanghai are municipalities, and the other seven are provincial capital cities: Harbin (Heilongjiang's provincial capital), Xi'an (Shaanxi), Nanjing (Jiangsu), Wuhan (Hubei), Guangzhou (Guangdong), Fuzhou (Fujian), and Kunming (Yunnan). Multistage stratified cluster sampling was used according to districts and kindergarten. In each city, one or more districts were selected as the study units, and those children aged 3-7 years, living in these study units represented the study subjects. Children at age 3 years or older attended kindergartens and started primary school at 7 years in China. The number of kindergartens was estimated based on the total number of children aged 3-7 years and their age distribution in the selected districts of each city, and the participation rate of the study subjects was not less than $95 \%$ in the selected kindergartens [12]. This study protocol was reviewed and approved by the Ethics Committees of the Capital Institute of Pediatrics. In the series of the NESSOC, consent was verbal, but there is not ethics issue. A written informed parental consent was presented at the head of the questionnaire. Before starting the questionnaire and the physical examination, the staffs of cooperating groups explained adequately the purposes and procedures of the surveys, the risk/discomforts and benefits to parents, guardians, and teachers who understand the potential risks and all the questions involved and voluntarily agree to participate in these surveys.

\section{Definition, screening and grouping of obesity}

Weight and height values of each child documented annually or semiannually through regular physical examination were taken as a crude screening basis in our studies. Current weight and height values were measured in the field sites of kindergartens for determining obesity using a weight-for-height $\geq 120 \%$ of median of NCSH/WHO [13]. Any extreme values caused by measurement errors could be easily picked out and corrected immediately according to the child's health record. Secondary or pathological obesity was identified by senior doctors according to "inclusion and exclusion criteria of childhood simple obesity" for separate statistics.

To examine risk factors, the population-based, 1:1 matched case-control design was employed in the series of survey. Due to the variation of potential risk factors among ages and sexes, one identified obese child was matched with one randomly selected non-obese child by sex, age (difference less than half a year) and height (difference within $\pm 3.0 \mathrm{~cm}$ ) from the same kindergartens.

In order to make results more likely to be compared, in the present analysis we redefined the obesity using the International Obesity Task Force (IOTF) BMI-forage cut-off points [14]. Obese children extracted by the
IOTF standards from original dataset and their corresponding non-obese children as the subjects of this study were showed in Fig. 2.

\section{Questionnaire}

The questionnaire included child status in early life, maternal condition during pregnancy, dietary habit, behavior and lifestyle, family information/environments. Set of questionnaire items appropriately adjusted in the series of surveys because of the social changes of China. Potential risk factors and confounders of obesity were chosen in the light of previously reported associations, or plausible prior hypotheses.

\section{Measurement and quality control}

Weight and height of all children were measured using unified measuring tools/instruments in a standardized way by specially trained technicians or nurses [15]. The weight was obtained with lever scale to the nearest $0.01 \mathrm{~kg}$ with children wearing the lightest vest, shorts or underwear. The height was measured on height board on level ground with bare feet to the nearest $0.1 \mathrm{~cm}$, the back of the head of child, shoulder blades, buttocks and heels all touch the vertical board and a horizontal line from the ear canal to the lower border of the eye socket runs parallel to the baseboard. Errors of weight and height were not more than $0.05 \mathrm{~kg}$ and $0.5 \mathrm{~cm}$ between two repeated measurements, respectively. Parents could be met in the kindergarten when they picked up their children. Questionnaires involving activity and feeding pattern of the child and parental information such as education level, attitude to obesity and their current weight and height were administered to the parents on the day of investigation. Part of the information about child daily physical activities was reported by kindergarten teachers. The study subgroups of each city collected and checked measurements and questionnaires under the direction of the Beijing Steering Committee that ultimately performed data entry and statistical analysis.

\section{Statistical analysis}

Potential risk factors of obesity were examined using univariate and multivariate conditional logistic regression models. Odds ratio (OR) more or less than $1.00(P<0.05)$ was considered to be a statistically identified risk factor. Potential interactions were explored between appetite and eating speed, and between outdoor activity and TV viewing, and between parental BMI and income of family. Data were analyzed with SAS version 9.2 (SAS Institute Inc., Cary, NC).

\section{Results}

As outlined in Fig. 2, of the 3294 and 5950 (obese and non-obese) participants based on a weight-for- 

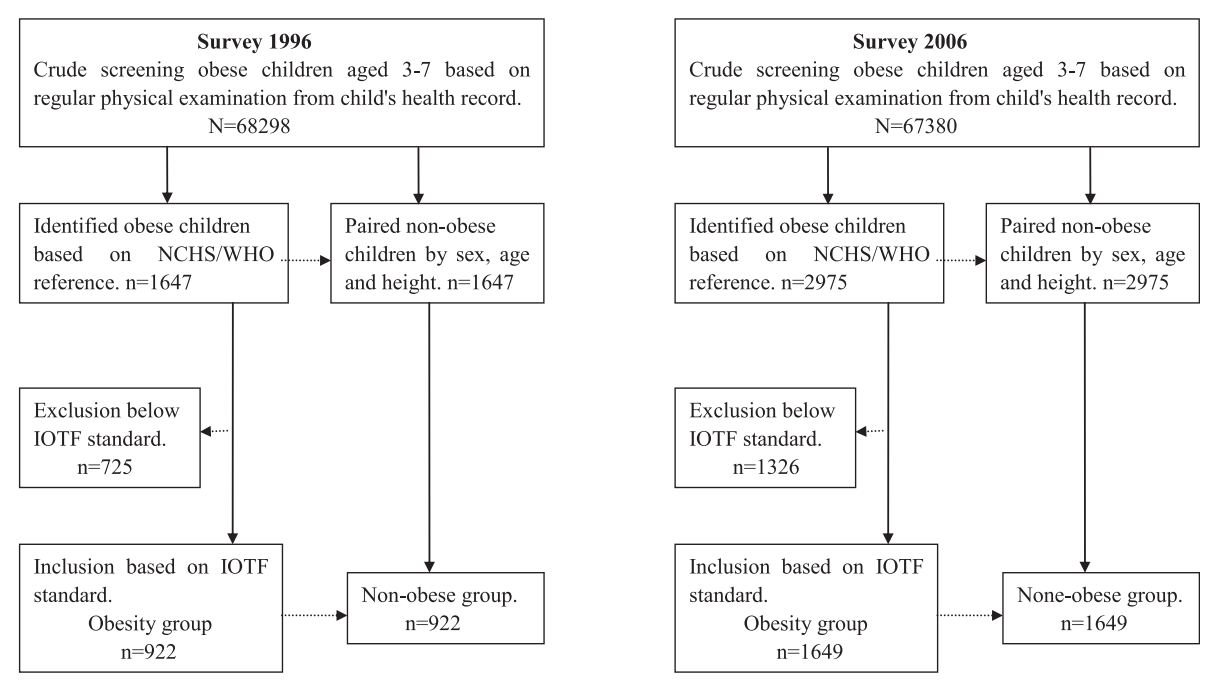

Fig. 2 Flow chart of the study population in 1996 and 2006 surveys

height $\geqq 120 \%$ of median of the NCHS/WHO reference in the 1996 and 2006 surveys, respectively. The corresponding 1844 and 3298 subjects were extracted in accordance with the IOTF BMI-for-age cut offs. The prevalence of obesity was $1.49 \%$ (boys $1.93 \%$ and girls $1.02 \%$ ) in 1996 and $2.64 \%$ (3.47 and $1.74 \%$ ) in 2006, with $0.12 \%$ point per year of increasing rate (boys 0.15 and girls 0.07 ). Table 1 presented sample sizes, height and BMI medians (I-III quartile) for obese children and the controls by sex and age in the 1996 and 2006. Comparison of the two groups seems to be not significant difference for height but obviously fatter for BMI in the obese than the controls.

\section{Intrauterine and perinatal factors}

Tables 2 and 3 showed high birth weight and gestational hypertension were associated with children obesity.
Gestational diabetes seemed to have no role in early obesity. From the feeding pattern in the first 4 months, breast feeding was a protective factor in univariate model in $2006(\mathrm{OR}=0.834, P=0.0234)$, but the association was not seen in multivariate.

\section{Dietary habit, behavior and lifestyle}

Appetite and eating speed presented significant positive correlations with obesity. Daily time and type/intensity for outdoor activities were positively connected with obesity in univariate analysis, with their associations of $\mathrm{OR}=$ $0.845(P=0.0247)$ and $0.725(P<0.0001)$ respectively, and outdoor activities (running or jumping) also entered multivariate model in 1996 and 2006. Associations of daily sleep time were $\mathrm{OR}=0.935(P=0.0033)$ in 1996 and 0.942 $(P=0.0035)$ in 2006 according to univariate analysis, and

Table 1 Sample sizes, median and quartile of height and BMI between obese and control groups in the 1996 and 2006 surveys

\begin{tabular}{|c|c|c|c|c|c|c|c|c|c|c|}
\hline \multirow{3}{*}{ Age (years) } & \multicolumn{5}{|c|}{ Boys } & \multicolumn{5}{|l|}{ Girls } \\
\hline & \multirow[t]{2}{*}{$\bar{n}$} & \multicolumn{2}{|c|}{ Height in $\mathrm{cm}(\mathrm{M} \pm \mathrm{Q})$} & \multicolumn{2}{|c|}{$\mathrm{BMl}$ in $\mathrm{kg} / \mathrm{m}^{2}(\mathrm{M} \pm \mathrm{Q})$} & \multirow[t]{2}{*}{$\bar{n}$} & \multicolumn{2}{|c|}{ Height in $\mathrm{cm}(\mathrm{M} \pm \mathrm{Q})$} & \multicolumn{2}{|c|}{ BMl in $\mathrm{kg} / \mathrm{m}^{2}(\mathrm{M} \pm \mathrm{Q})$} \\
\hline & & Obese & Control & Obese & Control & & Obese & Control & Obese & Control \\
\hline \multicolumn{11}{|l|}{ Survey 1996} \\
\hline $3-3.9$ & 76 & $103.3 \pm 7.8$ & $102.1 \pm 7.1$ & $20.91 \pm 2.07$ & $15.27 \pm 2.55$ & 52 & $104.0 \pm 6.7$ & $102.0 \pm 7.2$ & $20.29 \pm 1.99$ & $16.24 \pm 1.35$ \\
\hline $4-4.9$ & 182 & $111.5 \pm 6.1$ & $109.0 \pm 6.0$ & $20.45 \pm 1.83$ & $15.30 \pm 1.57$ & 106 & $109.2 \pm 5.1$ & $108.3 \pm 4.0$ & $20.26 \pm 1.41$ & $14.86 \pm 1.80$ \\
\hline $5-5.9$ & 434 & $117.5 \pm 6.6$ & $116.1 \pm 6.6$ & $20.79 \pm 2.37$ & $15.07 \pm 1.58$ & 232 & $116.0 \pm 6.3$ & $115.0 \pm 5.8$ & $20.32 \pm 1.85$ & $14.89 \pm 1.71$ \\
\hline $6-6.9$ & 542 & $123.0 \pm 5.8$ & $122.0 \pm 6.0$ & $21.31 \pm 2.08$ & $15.17 \pm 1.53$ & 220 & $122.5 \pm 6.2$ & $121.5 \pm 6.1$ & $21.35 \pm 1.57$ & $14.91 \pm 1.83$ \\
\hline \multicolumn{11}{|l|}{ Survey 2006} \\
\hline $3-3.9$ & 177 & $105.2 \pm 4.4$ & $104.5 \pm 6.6$ & $20.33 \pm 2.17$ & $15.93 \pm 1.18$ & 113 & $103.4 \pm 5.1$ & $101.4 \pm 6.1$ & $20.32 \pm 1.52$ & $15.34 \pm 1.30$ \\
\hline $4-4.9$ & 490 & $112.7 \pm 6.5$ & $111.0 \pm 5.6$ & $20.50 \pm 1.92$ & $15.35 \pm 1.38$ & 278 & $111.5 \pm 6.5$ & $109.3 \pm 6.1$ & $20.14 \pm 1.28$ & $15.15 \pm 1.40$ \\
\hline $5-5.9$ & 918 & $119.0 \pm 6.8$ & $117.4 \pm 5.6$ & $20.59 \pm 1.80$ & $15.37 \pm 1.43$ & 379 & $117.5 \pm 7.3$ & $116.5 \pm 6.9$ & $20.22 \pm 1.86$ & $15.14 \pm 1.54$ \\
\hline $6-6.9$ & 705 & $124.6 \pm 6.1$ & $123.5 \pm 5.8$ & $21.40 \pm 2.24$ & $15.47 \pm 1.59$ & 238 & $123.5 \pm 7.5$ & $122.1 \pm 5.2$ & $20.79 \pm 1.38$ & $14.96 \pm 1.41$ \\
\hline
\end{tabular}


Table $2 \mathrm{OR}$ and its $95 \% \mathrm{Cl}$ of some potential risk factors and confounders of obesity in preschool children using univariate conditional logistic regression

\begin{tabular}{|c|c|c|c|c|c|c|c|}
\hline \multirow[t]{2}{*}{ Variables } & \multirow[t]{2}{*}{ Factor level of analysis } & \multicolumn{3}{|c|}{ Survey 1996} & \multicolumn{3}{|c|}{ Survey 2006} \\
\hline & & $\mathrm{N}$ & OR $(95 \% \mathrm{Cl})$ & $P$ & $\mathrm{~N}$ & OR $(95 \%$ Cl) & P \\
\hline \multicolumn{8}{|l|}{ Intrauterine and perinatal factors } \\
\hline \multirow[t]{4}{*}{ Birth weight } & $<2500,2500-4000$ (ref.), $\geqq 4000$ & 1846 & & & 3176 & & \\
\hline & $2500-4000$ & 1556 & 1 & - & 2658 & 1 & - \\
\hline & $<2500$ & 31 & $1.060(0.508-2.212)$ & 0.8770 & 52 & $0.994(0.575-1.717)$ & 0.9815 \\
\hline & $\geqq 4000$ & 259 & $1.876(1.427-2.467)$ & $<0.0001$ & 466 & $1.880(1.525-2.319)$ & $<0.0001$ \\
\hline \multirow[t]{4}{*}{ Gestational age } & $<37,37-42$ (ref.), $\geqq 42$ & 1804 & & & 3244 & & \\
\hline & $37-42$ & 1644 & 1 & - & 2956 & 1 & - \\
\hline & $<37$ & 85 & $1.113(0.715-1.734)$ & 0.6354 & 134 & $1.214(0.853-1.730)$ & 0.2820 \\
\hline & $\geqq 42$ & 75 & $1.642(1.016-2.654)$ & 0.0436 & 154 & 1.117 (0.807-1.546) & 0.5030 \\
\hline No of fetuses & 1 (ref.), 2 (twins) & - & - & - & 3252 & $0.889(0.513-1.541)$ & 0.6750 \\
\hline Mode of delivery & Caesarean, vaginal (ref.) & - & - & - & 3234 & $1.381(1.195-1.595)$ & $<0.0001$ \\
\hline Gestational diabetes & No (ref.), yes & 1788 & $0.801(0.316-2.029)$ & 0.6374 & 3192 & $1.647(0.902-3.009)$ & 0.1011 \\
\hline Gestational hypertension & No (ref.), yes & 1798 & $1.838(1.231-2.743)$ & 0.0025 & 3200 & $2.392(1.539-3.717)$ & $<0.0001$ \\
\hline Feeding patterns in the first 4 months ${ }^{a}$ & Breast feeding, formula/milk (ref.) & 1846 & $0.917(0.731-1.150)$ & 0.4522 & 3200 & $0.834(0.713-0.976)$ & 0.0234 \\
\hline \multicolumn{8}{|l|}{ Dietary habit } \\
\hline \multirow[t]{4}{*}{ Appetite } & Good, average (ref.), bad & 1826 & & & 3236 & & \\
\hline & Average & 447 & 1 & - & 1102 & 1 & - \\
\hline & Good & 1335 & $14.326(9.651-21.266)$ & $<0.0001$ & 2074 & $10.995(8.622-14.02)$ & $<0.0001$ \\
\hline & $\mathrm{Bad}$ & 44 & $0.519(0.143-1.883)$ & 0.3123 & 60 & $0.209(0.072-0.602)$ & 0.0038 \\
\hline \multirow[t]{4}{*}{ Eating speed ${ }^{\mathrm{b}}$} & Fast, average (ref.), slow & 1820 & & & 3224 & & \\
\hline & Average & 1126 & 1 & - & 1674 & 1 & - \\
\hline & Fast & 444 & $8.282(5.739-11.952)$ & $<0.0001$ & 947 & $7.404(5.763-9.511)$ & $<0.0001$ \\
\hline & Slow & 250 & $0.136(0.083-0.222)$ & $<0.0001$ & 603 & $0.227(0.171-0.302)$ & $<0.0001$ \\
\hline Snack & Not at all, occasional, often & 1808 & $1.164(1.008-1.343)$ & 0.0380 & 3208 & $0.948(0.861-1.044)$ & 0.2789 \\
\hline Vegetable intake & Not at all, occasional, often & 1762 & $0.849(0.720-1.003)$ & 0.0532 & 3236 & $0.869(0.766-0.986)$ & 0.0292 \\
\hline \multicolumn{8}{|l|}{ Behavior and lifestyle } \\
\hline \multirow[t]{4}{*}{ Activity preferred } & Outdoor play, TV viewing (ref.), indoor game & 1194 & & & - & & \\
\hline & TV viewing & 484 & 1 & - & - & - & - \\
\hline & Outdoor play & 479 & $0.596(0.446-0.795)$ & $<0.0004$ & - & - & - \\
\hline & Indoor game & 231 & $0.876(0.629-1.221)$ & 0.4337 & - & - & - \\
\hline
\end{tabular}


Table $2 \mathrm{OR}$ and its $95 \% \mathrm{Cl}$ of some potential risk factors and confounders of obesity in preschool children using univariate conditional logistic regression (Continued)

\begin{tabular}{|c|c|c|c|c|c|c|c|}
\hline \multirow[t]{5}{*}{ Daily time for outdoor activity } & Time (hr): $<$ half (ref.), half-1, $1-2, \geqq 2$ & - & & & 3232 & & \\
\hline & $<$ half & - & - & - & 367 & 1 & - \\
\hline & Half-1 & - & - & - & 1143 & $0.955(0.753-1.212)$ & 0.5286 \\
\hline & $1-2$ & - & - & - & 1126 & $0.845(0.729-0.979)$ & 0.0247 \\
\hline & $\geqq 2$ & - & - & - & 596 & $1.019(0.773-1.343)$ & 0.8923 \\
\hline \multirow[t]{4}{*}{ Type/intensity of outdoor activity ${ }^{c}$} & Running or jumping, walking or cycling, slow walking or sitting (ref.) & 1212 & & & 3146 & & \\
\hline & slow walking or sitting & 148 & 1 & - & 808 & 1 & - \\
\hline & Running or jumping & 659 & $0.757(0.588-0.974)$ & 0.0302 & 2000 & $0.725(0.609-0.863)$ & $<0.0001$ \\
\hline & Walking or cycling & 405 & $0.674(0.351-1.294)$ & 0.1275 & 338 & $1.130(0.869-1.469)$ & 0.3601 \\
\hline \multirow[t]{5}{*}{ Daily time for TV viewing ${ }^{d}$} & Time (hr): $<$ half (ref.), half-1, $1-2, \geqq 2$ & - & & & 3184 & & \\
\hline & $<$ half & - & - & - & 447 & 1 & - \\
\hline & Half-1 & - & - & - & 1164 & $1.224(0.978-1.532)$ & 0.0776 \\
\hline & $1-2$ & - & - & - & 1117 & $1.373(1.171-1.610)$ & $<0.0001$ \\
\hline & $\geqq 2$ & - & - & - & 456 & $1.996(1.602-2.487)$ & $<0.0001$ \\
\hline Daily sleep time. & Unit $0.5 \mathrm{~h}$ & 1806 & $0.935(0.894-0.978)$ & 0.0033 & 3198 & $0.942(0.906-0.981)$ & 0.0035 \\
\hline Night sleep time & Unit $0.5 \mathrm{~h}$ & - & - & - & 3198 & $0.890(0.846-0.936)$ & $<0.0001$ \\
\hline \multicolumn{8}{|l|}{ Parents and family-related factors } \\
\hline \multirow[t]{4}{*}{ Family type ${ }^{e}$} & Nuclear family (ref.), extended, single-parent & - & & & 3242 & & \\
\hline & Nuclear family & - & - & - & 1788 & 1 & - \\
\hline & Extended & - & - & - & 1401 & $1.241(1.075-1.433)$ & 0.0021 \\
\hline & Single-parent & - & - & - & 53 & $0.756(0.426-1.341)$ & 0.3372 \\
\hline \multirow[t]{4}{*}{ Raising kids mainly by } & Parents (ref.), grandparents, or nannies/nursery & 1622 & & & 3250 & & \\
\hline & Parents & 1035 & 1 & - & 2427 & 1 & - \\
\hline & Grandparents & 399 & $1.277(1.010-1.614)$ & 0.0016 & 702 & $1.408(1.181-1.678)$ & 0.0002 \\
\hline & Nannies/nursery & 188 & $0.606(0.287-1.279)$ & 0.1842 & 121 & $1.308(0.779-2.194)$ & 0.0818 \\
\hline Mother education & College and over, high school and below (ref.) & 1794 & $0.851(0.667-1.086)$ & 0.1936 & 3242 & $0.731(0.625-0.855)$ & $<0.0001$ \\
\hline Father education & College and over, high school and below (ref.) & 1798 & $0.674(0.521-0.871)$ & 0.0025 & 3222 & $0.675(0.575-0.791)$ & $<0.0001$ \\
\hline \multirow[t]{4}{*}{ Family income in local } & Upper, middle (ref.), lower & 1828 & & & 3130 & & \\
\hline & Middle & 1559 & 1 & - & 2968 & 1 & - \\
\hline & Upper & 232 & $1.040(0.772-1.402)$ & 0.7955 & 85 & $1.546(0.967-2.473)$ & 0.0668 \\
\hline & Lower & 37 & $1.317(0.687-2.524)$ & 0.4111 & 77 & 1.948 (1.223-3.103) & 0.0049 \\
\hline
\end{tabular}


Table $2 \mathrm{OR}$ and its $95 \% \mathrm{Cl}$ of some potential risk factors and confounders of obesity in preschool children using univariate conditional logistic regression (Continued)

\begin{tabular}{|c|c|c|c|c|c|c|c|}
\hline \multirow[t]{4}{*}{ Family income per month } & RMB: $<1500,1500-10,000$ (ref.), $\geqq 10,000$ & - & & & 3100 & & \\
\hline & $1500-10,000$ & - & - & - & 2683 & 1 & - \\
\hline & $<1500$ & - & - & - & 189 & $1.435(1.042-1.977)$ & 0.0260 \\
\hline & $\geqq 10,000$ & - & - & - & 228 & $1.430(1.076-1.901)$ & 0.0152 \\
\hline Father BMI ${ }^{f}$ & $<24$ (ref.), $\geqq 24$ & 1804 & $2.525(2.041-3.125)$ & $<0.0001$ & 3152 & $2.164(1.866-2.510)$ & $<0.0001$ \\
\hline Mother BMI ${ }^{f}$ & $<24$ (ref.), $\geqq 24$ & 1790 & $4.031(3.072-5.288)$ & $<0.0001$ & 3192 & $2.538(2.075-3.104)$ & $<0.0001$ \\
\hline Mother family history of obesity ${ }^{9}$ & No (ref.), yes & 1826 & $2.644(2.110-3.313)$ & $<0.0001$ & - & - & - \\
\hline Father family history of obesity ${ }^{9}$ & No (ref.), yes & 1816 & $2.640(2.122-3.284)$ & $<0.0001$ & - & - & - \\
\hline
\end{tabular}

BMI Body mass index, OR Odds ratio, Cl Confidence interval

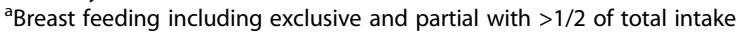

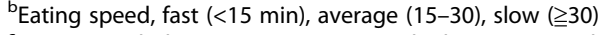

${ }^{\mathrm{C}}$ Running including running, jumping, climbing stair, et al.; sitting meaning not or hardly playing when others did

dTime for TV viewing including video game in 2006

'Parents-child based nuclear family, three generations based (including grandparents) extended, single-parent based

$\mathrm{f}_{\mathrm{BMI}}$ calculated as weight $(\mathrm{kg})$ divided by height $(\mathrm{m})$ squared based on self-reported weight and height, and overweight in Chinese adults was defined using the cutoffs of $24 \mathrm{~kg} / \mathrm{m}^{2}$

${ }^{9}$ Obesity history among three generations of parents, including their grandparents, their parents and their siblings, brothers and sisters 
Table 3 Associations between risk factors and obesity in preschool children using multivariate conditional logistic regression

\begin{tabular}{|c|c|c|c|c|c|}
\hline \multirow[t]{2}{*}{ Variables } & \multirow[t]{2}{*}{ Factor level of analysis } & \multicolumn{2}{|l|}{ Survey 1996} & \multicolumn{2}{|l|}{ Survey 2006} \\
\hline & & OR $(95 \%$ Cl) & $P$ & OR $(95 \% \mathrm{Cl})$ & $P$ \\
\hline \multirow[t]{2}{*}{ Birth weight } & $<2500,2500-4000$ (ref.), $\geqq 4000$ & & & & \\
\hline & $\geqq 4000$ & - & - & 1.609 (1.124-2.303) & 0.0094 \\
\hline Gestational hypertension & No (ref.), yes & $20.538(3.453-122.698)$ & 0.0009 & $2.412(1.029-5.653)$ & 0.0428 \\
\hline \multirow[t]{2}{*}{ Appetite } & Good, average (ref.), bad & & & & \\
\hline & Good & $12.433(5.562-27.793)$ & $<0.0001$ & $6.266(4.521-8.686))$ & $<0.0001$ \\
\hline \multirow[t]{3}{*}{ Eating speed ${ }^{\mathrm{a}}$} & Fast, average (ref.), slow & & & & \\
\hline & Fast & $8.039(3.599-17.960)$ & $<0.0001$ & $4.351(3.131-6.046)$ & $<0.0001$ \\
\hline & Slow & $0.173(0.054-0.555)$ & 0.0032 & $0.343(0.225-0.522)$ & $<0.0001$ \\
\hline Vegetable intake & Not at all, occasional, often & $0.533(0.334-0.850)$ & 0.0082 & - & - \\
\hline \multirow[t]{2}{*}{ Type/intensity of outdoor activities ${ }^{b}$} & Running or jumping, walking or cycling, slow walking or sitting (ref.) & & & & \\
\hline & Running or jumping & $0.191(0.066-0.847)$ & 0.0022 & $0.595(0.445-0.795)$ & 0.0004 \\
\hline \multirow[t]{3}{*}{ Daily time for TV viewing ${ }^{c}$} & Time (hr): $<$ half (ref.), half-1, $1-2, \geqq 2$ & & & & \\
\hline & $1-2$ & - & - & $1.339(1.003-1.789)$ & 0.0477 \\
\hline & $\geqq 2$ & - & - & $2.154(1.455-3.188)$ & $<0.0001$ \\
\hline Father education & College and over, high school and below (ref.) & - & - & $0.661(0.494-0.886)$ & 0.0056 \\
\hline Father BMI $^{d}$ & $<24$ (ref.), $\geqq 24$ & $2.326(1.279-4.232)$ & 0.0057 & $2.124(1.634-2.762)$ & $<0.0001$ \\
\hline Mother BMI $^{d}$ & $<24$ (ref.), $\geqq 24$ & $2.495(1.307-4.761)$ & 0.0056 & $1.529(1.089-2.146)$ & 0.0142 \\
\hline \multirow[t]{2}{*}{ Raising kids mainly by } & Parents (ref.), grandparents, nannies & & & & \\
\hline & Grandparents & - & - & $1.439(1.050-1.973)$ & 0.0236 \\
\hline Father family history of obesity ${ }^{\mathrm{e}}$ & No (ref.), yes & $1.889(1.045-3.414)$ & 0.0352 & - & - \\
\hline
\end{tabular}

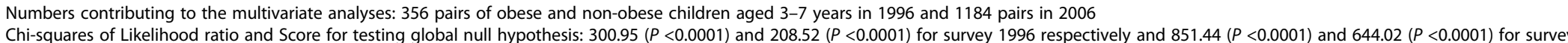
2006 respectively

$B M I$ Body mass index, $O R$ Odds ratio, $C I$ Confidence interval

aEating speed, fast ( $<15 \mathrm{~min})$ average $(15-30)$, slow $(\geq 30)$

bRunning including running jumping, climbing stair, et al: sitting meaning not or hardly playing when others did

'Time for TV viewing including video game in 2006 '

${ }^{d}$ BMI calculated as weight $(\mathrm{kg})$ divided by height $(\mathrm{m})$ squared based on self-reported weight and height, and overweight in Chinese adults was defined using the cutoffs of $24 \mathrm{~kg} / \mathrm{m}^{2}$

eObesity history among three generations of parents, including their grandparents, their parents and their siblings, brothers and sisters 
protective effect of night sleep time was $\mathrm{OR}=0.890$ $(P<0.0001)$ in 2006 . Daily time for TV viewing was closely related to obesity, with $\mathrm{OR}=1.339(P=0.0477)$ for $1-2 \mathrm{~h}$ level and 2.154 $(P<0.0001)$ for $\geqq 2 \mathrm{~h}$ level in multivariate model in 2006 (Tables 2 and 3).

\section{Family environment}

Compared with parents based nuclear family, those children brought up in extended family (including grandparents) might be at an increased risk of becoming obese. Those children mainly raised by their grandparents might be more susceptible to obesity than those looked after by parents. Higher or lower income families might have a negative effect on weight gain of children, and high income family ultimately entered multivariate logistic regression (Tables 2 and 3).

\section{Parents' BMI}

Association of children obesity with their parents' BMI was repeatedly identified in the 1996 and 2006. In addition, the influence of mother and father family history of obesity on children obesity was also observed in the 1996 (Tables 2 and 3).

\section{Parents' education and attitude}

Parents' education at college and over might be a protective factor for children obesity, and father education was further screened out by multivariate model in the 2006. In the 1996, $15.7 \%$ parents were not aware of the obesity of their children and $30.0 \%$ parents did not control food and diet intakes for their children in obesity group; only $8.9 \%$ parents helped their children select food in comparison with the proportion of $27.9 \%$ in the controls $(P<0.01)$. In the 2006, still $11.2 \%$ parents didn't realize the obese status of their children for the obesity, and $18.7 \%$ parents believed obesity was a good thing for boys in the obese and the proportion was $14.7 \%(P<0.01)$ in the controls.

\section{Discussion}

Not only is obesity increasing worldwide, but no national success stories in obesity prevention and control have been reported over the past decades [16]. Obesity epidemics driven by complex forces require system thinking to conceptualise the causes and to organise evidence needed for action [17]. To the authors' knowledge, obesity prevention and control for preschool children was not yet paid enough attention in comparison of adults and teenagers. To some extent, data sets of risk factors for childhood obesity are not well established because of the difference of lifestyles, behaviors and social cultures among different nations, countries and regions. Some highly suspected risk factors associated with preschool children obesity have not yet integrated systematically an effective force to counter rapidly rising obesity rates.
Childhood obesity prevention research must now move towards identifying how effective intervention components can be embedded within health, education and care systems and achieve long term sustainable impacts [18]. In this study a wider spectrum of risk factors and an empirical aggregation of family-related risk factors are discussed to further improve future family-based child obesity prevention and control strategies.

Genetic factors and intrauterine/perinatal status may influence the susceptibility of individuals to weight gain. The percentage of obesity that could be attributed to genetics varies from 6 to $85 \%$ depending on examined population [19]. Our study demonstrated parental obesity and maternal/paternal obesity affected the weight status of the offsprings which were also observed in rural China [20]. High birth weight and gestational hypertension were considered to be at high risk of future obesity, suggesting the importance of early environments from pregnancy and infancy. Increased risk of birth weight and maternal BMI on early childhood obesity was also confirmed by a cohort study from China [21]. These evidence was consistent with a cohort study on early life risk factors for obesity in childhood in the United Kingdom [22].

Dietary changes towards higher in fats and calories and increasing physical inactivity have been driving progressive increase of weight and epidemic of obesity among Chinese children after the economic transition [23]. Preschool children in Chinese high-income families started to be exposed to those foods with high calorie and high fat, such as Western fast food, fried foods, sweet drinks and so on. Perhaps there are also such some sedentary lifestyles for children in this age as watching TV viewing/video games and taking training courses of outside kindergarten (e.g. foreign languages, drawing and piano). One study from Bangladesh showed limited exercise and high levels of sedentary activities along with having overweight parents more often lead to obesity [24].

There is consensus that changes in lifestyle behaviors are driving the obesity epidemic [25]. Families and parents have profound influence on obesity-related behaviors of their children. Current review has emphasized the role of the home and environment in affecting health behaviors in children and adolescents at high risk for developing chronic disease [3]. Despite observational evidence for the role of parenting in children's weight-related outcomes emerged, few interventions have been developed that address general parenting in childhood obesity prevention [26]. Our study showed children who were mainly raised by their grandparents were more likely to suffer from obesity, which was also seen in another study from China [27]. Children living in low educational background of parents or families were more likely to be obesity because of a shortage of the knowledge of food selection, energy 
balance and weight control. Many in China have viewed plumpness as a sign of prosperity and healthy development and accepted the perception "boys should be stronger and bigger". And because of the impact of the one-child policy, only one child in a family was usually doted upon by their parents and grandparents who are almost given all the treats available with a rising income [28]. Sex difference, higher prevalence of obesity in Chinese boys than girls, reflected the potential impact of ethnicity and socioculture of different countries or regions. So the perspective about the importance of ethnicity and culture should be taken into consideration [29]. Childhood is an important period of a person for the shape of behaviors, habits and lifestyles which mostly originated from their parents and families. The impact of families on childhood obesity is strongly supported, implying the value of family interventions involving obesity-related behaviors. One recent study concluded the effectiveness of family-based behavioral multi-component intervention for the treatment of overweight and obese children [30].

Most of the risk factors identified by this study presented ranked or quantitative characteristics which might be transformed from unhealthy threshold to healthy range by behavior modification. The present work paves a path for further revising or developing family-based child obesity prevention programs. Future suggesting measures might focus on the amelioration of eating behavior, physical activity, sleep and TV viewing on all fronts to prevent the occurrence of obesity. Our study is in agreement with three major obesity-related behaviors, i.e. dietary intake, physical activity and sleep [31]. Parents played the leading roles in their sharing milieu, and their healthy lifestyles or behavioral habits could be infiltrated to their children. Children in this age are usually enrolled in kindergartens. Earlier studies have indicated that school and family environments are key determinants of energy-balance behaviors in school children. Therefore, kindergartens and families should collaborate with each other to create a better growth environment for the well-being of children.

Some variables are likely to interact each other, such as appetite and eating speed, or outdoor activity and TV viewing, or BMI and income. According to the results of univariate analysis for 2006 data, the interaction between appetite and eating speed was significant for the level of fast eating speed and good appetite, with $\mathrm{OR}=30.167$ $(P<0.0001,95 \%$ CI (19.962-45.588)), average level as control; the interaction of time of outdoor activity and time of $\mathrm{TV}$ viewing (as continuous variable) was significant with $\mathrm{OR}=1.041 \quad(P=0.0015,95 \% \mathrm{CI}(1.016-1.067))$; the interactions of income of family and father obesity were significant with $\mathrm{OR}=1.479(P<0.0001,95 \% \mathrm{CI}$ $(1.356-1.612))$ and with mother obesity with $\mathrm{OR}=1.522$ $(P<0.0001,95 \%$ CI (1.354-1.710)). However, these interactions of the aforementioned factors could not enter the multivariate models at the 0.05 level of $p$ value, perhaps their effects need to be further explored in future surveys.

\section{Conclusions}

Health depends more on living conditions, behavior lifestyle and guarantee of medical expenses and the early prevention and control for excessive weight gain is often therefore considered as the most effective way to reduce the risk of overweight and obesity. Although some risk factors identified by this study were also reported in other studies, we observed obvious characteristics of family clustering of risk factors of obesity in the earlier stage of life. A majority of our identified influence factors could be summarized an empirical aggregation of family-related risk factors involving family heredity history, fetal birth weight, parents' attitude to obesity, eating behavior, physical activity, TV viewing, and raising kids by grandparents. Family-related risk factors, as an integrated concept, would be helpful to promote future family-based prevention and control programs, early detect potential high-risk individuals in early life and enable provision of flexible and personalized interventions based on personal behaviors and needs of the child. According to our results, the family-related risk factors strongly supported the further development of family-based programs in the preschool period. Our study would also add to mounting evidence for future suggesting measures in the process of family-based obesity intervention programs in childhood.

\section{Abbreviations \\ BMl: Body mass index; OR: Odds ratio; Cl: Confidence interval; NCHS/ WHO: National Center for Health Statistics/World Health Organization; IOTF: International Obesity Task Force.}

Competing interests

The authors declare that they have no competing interests.

\section{Authors' contributions}

Conceived and designed the surveys: HL. Analyzed the data and wrote the first draft: XNZ. Interpreted and discussed the results: XNZ, HL, YQZ. All authors read and approved the final version.

Authors' information

Not applicable

Availability of data and materials

Not applicable

\section{Acknowledgements}

The series of the NESSOC work was supported by the Ministry of Health of the People's Republic of China. We thank all preschool children and their parents and teachers participating in this study. We thank the technical support of all the team members in the NESSOC. We thank the three reviewers, Dario Gregori, Emanuela Gualdi and Stefania Toselli for their insightful comments.

\section{Funding}

The publishing fee was supported by the National Natural Science Foundation of China (No.81302439) which had no role in the design and data collection of the NESSOC. 


\section{Disclaimer}

The content is solely the responsibility of the authors and does not necessarily represent the official views of the Ministry of Health of the People's Republic of China.

Received: 20 June 2014 Accepted: 13 September 2015 Published online: 19 September 2015

\section{References}

1. de Onis M, Blössner M, Elaine B. Global prevalence and trends of overweight and obesity among preschool children. Am J Clin Nutr. 2010;92:1257-64

2. Yu Z, Han S, Chu J, Xu Z, Zhu C, Guo X. Trends in overweight and obesity among children and adolescents in China from 1981 to 2010: a meta-analysis. PLoS One. 2012;7:e51949.

3. Lawman $\mathrm{HG}$, Wilson DK. A review of family and environmental correlates of health behaviors in high-risk youth. Obesity (Silver Spring). 2012;20:1142-57.

4. Kitzman-Ulrich H, Wilson DK, St George SM, Lawman H, Segal M, Fairchild A. The integration of a family systems approach for understanding youth obesity, physical activity, and dietary programs. Clin Child Fam Psychol Rev. 2010;13:231-53.

5. Dobbins M, Husson H, DeCorby K, LaRocca RL. School-based physical activity programs for promoting physical activity and fitness in children and adolescents aged 6 to 18. Cochrane Database Syst Rev. 2013;2:CD007651.

6. Katz DL, O'Connell M, Njike VY, Yeh MC, Nawaz H. Strategies for the prevention and control of obesity in the school setting: systematic review and meta-analysis. Int J Obes (Lond). 2008:32:1780-9.

7. Sharma M. International school-based interventions for preventing obesity in children. Obes Rev. 2007:8:155-67.

8. Brug J, te Velde SJ, Chinapaw MJ, Bere E, de Bourdeaudhuij I, Moore H, et al. Evidence-based development of school-based and family-involved prevention of overweight across Europe: the ENERGY-project's design and conceptual framework. BMC Public Health. 2010;10:276.

9. Showell NN, Fawole O, Segal J, Wilson RF, Cheskin L, Bleich SN, et al. A systematic review of home-based childhood obesity prevention studies. Pediatrics. 2013;132:e193-200.

10. Wang $H$, Zhai F. Programme and policy options for preventing obesity in China. Obes Rev. 2013;14 Suppl 2:134-40.

11. Zong X-N, Li H. Secular trends in prevalence and risk factors of obesity in infants and preschool children in 9 Chinese cities, 1986-2006. PLoS One. 2012;7:e46942

12. Li H, Coordinating Group of Nine Cities Study on the Physical Growth and Development of Children, Capital Institute of Pediatrics. A national epidemiological survey on obesity of children under 7 years of age in nine cities of China, 2006. Zhonghua Er Ke Za Zhi. 2008:46:174-8.

13. World Health Organization. Physical status: the use and interpretation of anthropometry. Report of a WHO Expert Committee. World Health Organ Tech Rep Ser. 1995:854:1-452.

14. Cole TJ, Bellizzi MC, Flegal KM, Dietz WH. Establishing a standard definition for child overweight and obesity worldwide: international survey. BMJ. 2000;320:1240-3.

15. Li H, Coordinating Study Group of Nine Cities on the Physical and Development of Children, Capital Institute of Pediatrics. The national growth survey of children under 7 years in the nine cities of China. Beijing: People's Medical Publishing House; 2008.

16. Ng M, Fleming T, Robinson M, Thomson B, Graetz N, Margono C. Global, regional, and national prevalence of overweight and obesity in children and adults during 1980-2013: a systematic analysis for the Global Burden of Disease Study 2013. Lancet. 2014;384(9945):766-81.

17. Gortmaker SL, Swinburn BA, Levy D, Carter R, Mabry PL, Finegood DT, et al. Changing the future of obesity: science, policy, and action. Lancet. 2011;378:838-47.

18. Castro AM S e. Interventions for preventing obesity in children. Sao Paulo Med J. 2014;132:128-9.

19. Yang W, Kelly T, He J. Genetic epidemiology of obesity. Epidemiol Rev. 2007;29:49-61

20. Guo X, Zheng L, Li Y, Zhang X, Yu S, Yang H, et al. Prevalence and risk factors of being overweight or obese among children and adolescents in northeast China. Pediatr Res. 2013;74:443-9.
21. Ye R, Pei L, Ren A, Zhang Y, Zheng X, Liu JM. Birth weight, maternal body mass index, and early childhood growth: a prospective birth cohort study in China. J Epidemiol. 2010;20:421-8.

22. Reilly JJ, Armstrong J, Dorosty AR, Emmett PM, Ness A, Rogers I, et al. Early life risk factors for obesity in childhood: cohort study. BMJ. 2005;330:1357.

23. Zong X-N, Li H. Physical growth of children and adolescents in China over the past 35 years. Bull World Health Organ. 2014;92:555-64.

24. Bhuiyan MU, Zaman S, Ahmed T. Risk factors associated with overweight and obesity among urban school children and adolescents in Bangladesh: a case-control study. BMC Pediatr. 2013;13:72.

25. Hill JO, Wyatt HR, Reed GW, Peters JC. Obesity and the environment: where do we go from here? Science. 2003;299:853-5.

26. Gerards SM, Sleddens EF, Dagnelie PC, de Vries NK, Kremers SP. Interventions addressing general parenting to prevent or treat childhood obesity. Int J Pediatr Obes. 2011;6:e28-45.

27. Li B, Adab P, Cheng KK. Family and neighborhood correlates of overweight and obesogenic behaviors among Chinese children. Int J Behav Med. 2014;21:700-9.

28. Lobstein T. China joins the fatter nations. Int J Pediatr Obes. 2010;5:362-4.

29. Peña MM, Dixon B, Taveras EM. Are you talking to ME? The importance of ethnicity and culture in childhood obesity prevention and management Child Obes. 2012;8:23-7.

30. Serra-Paya N, Ensenyat A, Real J, Castro-Viñuales I, Zapata A, Galindo G, et al. Evaluation of a family intervention programme for the treatment of overweight and obese children (Nereu Programme): a randomized clinical trial study protocol. BMC Public Health. 2013;13:1000.

31. Spruijt-Metz D. Etiology, treatment and prevention of obesity in childhood and adolescence: a decade in review. J Res Adolesc. 2011;21:129-52.

\section{Submit your next manuscript to BioMed Central and take full advantage of:}

- Convenient online submission

- Thorough peer review

- No space constraints or color figure charges

- Immediate publication on acceptance

- Inclusion in PubMed, CAS, Scopus and Google Scholar

- Research which is freely available for redistribution 